\title{
Impact of school vaccination mandates on pediatric vaccination coverage: a systematic review
}

\author{
Devon Greyson PhD MLIS, Chris Vriesema-Magnuson MLIS, Julie A. Bettinger PhD MPH
}

\section{Abstract}

Background: Mandated vaccination for school attendance is a growing strategy internationally. Our aim was to investigate the effects of implementing school vaccination mandates on pediatric population vaccine coverage.

Methods: In this systematic review, we searched MEDLINE, Embase, CINAHL, the Education Resources Information Center (ERIC) and the PAIS Index for empirical studies of implementation of a primary or secondary school vaccination requirement published in any language through March 2019 with vaccination rates as an outcome. We sought additional studies by consulting experts, reference lists and grey literature sources. Included studies were too heterogeneous for meta-analysis; thus, we extracted data using a standardized rubric and synthesized the results narratively.

Results: Among the 4232 citations obtained, 20 studies met the inclusion criteria. Eighteen were conducted with US data, 1 with Italian data, and 1 with Australian data. Four studies examined school-entry mandates, and 16 examined adolescent requirements. An uncontrolled before-after design was used in 10 studies, cross-sectional analysis in 7, a retrospective cohort design in 2 , and a prospective cohort in 1. In many cases, increased documentation of coverage followed the addition of new requirements. The exception to this was human papillomavirus vaccination mandates, which were highly controversial, in the United States. The studies contained notable risks of bias, with cointerventions rarely acknowledged or accounted for, and subpopulations often excluded. A substantial risk of ecological fallacy existed for most studies.

Interpretation: Vaccination mandates appear largely associated with increased vaccination coverage, but it is not possible to attribute causality to the mandate in most studies. High-quality implementation research that uses whole-population coverage data and takes into consideration cointerventions, confounders, clustering of unvaccinated populations and context is required.

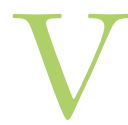
accination mandates for school attendance are ubiquitous in the United States and are growing as a strategy in Europe and Australia. Although the Canadian Medical Association passed a resolution in 2015 recommending that "governments authorize elementary and secondary schools to require a declaration of immunization status, to be followed by a conversation between public health officials and parents where children are shown to be inadequately immunized," 1 school vaccination legislation currently exists in just 2 Canadian provinces, Ontario ${ }^{2}$ and New Brunswick, ${ }^{3}$ with intent to implement a policy in British Columbia recently announced. ${ }^{4}$ Mandate policies vary in requirements (documentation, education, vaccination), schedule (which vaccines, when), restrictiveness (exemption processes), ${ }^{5}$ and incentives (e.g., financial rewards) or penalties (e.g., fines, school exclusion). Debate regarding best practices for mandate policies tends to draw largely on ethical arguments ${ }^{6}$ regarding the optimal legislation to achieve maximum pediatric vaccine coverage with minimal violation of parental civil liberties, with some voices advocating strict policies permitting few exemptions, others favouring a more libertarian approach, and many aiming to strike a balance. ${ }^{7}$ Often in these debates, the effectiveness of vaccination mandate laws in increasing population vaccine coverage is assumed; however, recent systematic assessment of the literature regarding the impacts of school mandates on vaccine coverage in Canada and other wealthy countries has not, to our knowledge, been done.

To assess the effectiveness of school vaccination mandates in real-life settings, studies with appropriate methods and outcomes must be examined. Cross-sectional studies have documented associations between the existence (or strictness) of a

Competing interests: Devon Greyson reports grants from the British Columbia Immunization Committee during the conduct of the study. No other competing interests were declared.

This article has been peer reviewed.

Correspondence to: Devon Greyson, dgreyson@umass.edu CMAJ Open 2019. DOI:10.9778/cmajo.20180191 
child vaccination mandate and population coverage, suggesting that restrictive policies around exemptions from vaccination mandates may increase compliance. ${ }^{8-11}$ These studies alone, however, cannot determine causality and are vulnerable to ecological biases. Other studies have explored the influence of mandates on outcomes such as exemption rate ${ }^{12,13}$ and disease occurrence, ${ }^{14}$ but disease is affected by many factors including temporal trends, and overall exemptions may mask clustering of unvaccinated populations that may raise the risk of disease outbreak. A focused systematic review comparing studies assessing the preferred outcome of population vaccination coverage is required to assess evidence for or against implementing new vaccination mandate policies. Our aim in this analysis was to inform the ongoing discussion regarding optimal childhood vaccination policy by systematically identifying and synthesizing the existing evidence to answer the question: What is the impact of implementation of school vaccination mandate policies on school-age population vaccine coverage?

\section{Methods}

\section{Research design}

This systematic review with narrative synthesis is reported according to PRISMA guidelines. ${ }^{15}$ In designing and implementing this review, we referred to the Cochrane Handbook for Systematic Reviews of Interventions ${ }^{16}$ for guidance. The protocol for this nonclinical review of policy interventions was not registered.

\section{Selection criteria}

We sought studies published in any language, using any empirical method, to obtain evidence on the effects of implementation of school vaccination mandates on the outcome of population vaccine coverage. Appropriate comparison groups included same-time comparators in locations without mandate changes or pre-post intervention comparisons. Studies that focused on individual school rules rather than regional/ government policy, mandates for nonpediatric or nonschool populations, or outbreak-specific policies were excluded, as were nonempirical papers and studies that examined only outcomes other than population vaccine coverage.

\section{Data collection/search strategy}

Two of the authors (C.V.-M. and D.G.) searched MEDLINE, Embase, CINAHL, the Education Resources Information Center (ERIC) and the PAIS Index in March 2019 to identify potentially relevant articles (search details provided in Appendix 1, available at www.cmajopen.ca/content/7/3/E524/suppl/ DC1). Searches combined database-specific subject headings and keywords for the concepts vaccination, law/policy and schools. Our MEDLINE search strategy was peer reviewed through Peer Review of Electronic Search Strategies (PRESS). ${ }^{17}$ We conducted limited searches for grey literature in thesis and dissertation databases, and databases that include conference abstracts and working papers (Appendix 1). References were searched and experts consulted to identify studies, including grey literature, missed by database searching.

\section{Screening and abstraction}

Titles and abstracts were independently screened in duplicate by 2 authors (C.V.-M. and D.G.) for relevance. Full texts of potentially includable articles were obtained, and all of the authors reviewed them independently in triplicate. Discrepancies were resolved by discussion among the authors to reach consensus. Included articles were then subjected to a data-extraction process independently in duplicate by 2 authors (D.G. and J.A.B.) (see Appendix 2, available at www.cmajopen.ca/content/7/3/E524/suppl/DC1, for data extraction fields).

\section{Risk of bias}

Although we were unable to find a tool to assess the risk of bias that directly applied to all the included studies, 2 authors (J.A.B. and D.G.) independently and in duplicate assessed each included study for potential bias in methods using the bias categories from the ROBINS-I (Risk Of Bias In Nonrandomised Studies - of Interventions) tool, ${ }^{18}$ adapted for relevance to this specific body of literature.

\section{Data analysis}

We synthesized the findings in a narrative manner using methods influenced by Popay and colleagues. ${ }^{19}$ Two authors (J.A.B. and D.G.) developed a preliminary textual synthesis of the characteristics and findings of the included studies, based on the tabulated extracted data. In an iterative manner, we explored relations within these data, grouping studies to examine the influence of heterogeneity (e.g., when looking by vaccine, target age group, location, data source, study design or type of mandate) on outcomes due to policy or setting details. We then incorporated our data on assessment of risk of bias into the descriptive synthesis of the included evidence to describe the robustness of the findings and temper the weight of the conclusions.

\section{Ethics approval}

As this study was solely literature based, it was not eligible for institutional ethics approval, and none was sought.

\section{Results}

Database searches resulted in 4232 unique citations to screen and assess for eligibility, and consulting experts and reference lists revealed 2 additional studies. After screening for relevance and applying inclusion and exclusion criteria, we selected 20 studies for inclusion in the review (Figure 1).

\section{Heterogeneity}

Of the 20 included studies, 18 were conducted with US data, 1 was from Italy, ${ }^{20}$ and 1 was from Australia. ${ }^{21}$ In 4 studies, the investigators looked at school-entry mandates implemented from 1970 to $2017,{ }^{20-22}$ and the remaining 16 (all set in the US) examined adolescent mandates (sometimes referred to as "middle school" requirements) from the 1990 s to $2015 .^{23-38}$ An uncontrolled before-after design was used in 10 studies, cross-sectional analysis in 7 and retrospective 
cohort design in 2, and 1 study was a prospective cohort study. Population size ranged from $583^{23}$ to $954973,{ }^{22}$ and time frames ranged from single cross-sectional surveys ${ }^{27,31,38}$ to a 17 -year span. ${ }^{22}$ Types of data included parental report, administrative data sets and clinician-verified records, and sources included various iterations of the US National Immunization Survey-Teen, ${ }^{24,25,30,33,34,37}$ state/national vaccine registries, ${ }^{20,22,35,36}$ school immunization databases, ${ }^{21,28,29}$ clinical registries, ${ }^{26,32}$ clinic and school sampling, ${ }^{27,38,39}$ randomdigit-dial survey ${ }^{23}$ and the US Health Plan Employer Data and Information Set. ${ }^{31}$ The most commonly used data source, the US National Immunization Survey-Teen, is a random-digit-dial telephone survey of parents of adolescents aged 13-17 years in 50 US states and the District of Columbia that been conducted since 2006. Most of the included studies assessed coverage following the addition of new vac- cines in a setting with existing mandates (e.g., addition of an adolescent pertussis vaccine dose), although 1 examined a new documentation mandate, ${ }^{21} 1$ examined a tightened exemption procedure, ${ }^{22} 2$ included education mandates, ${ }^{24,33}$ and 1 reported on added vaccines combined with stricter enforcement. ${ }^{20}$ Table 1 summarizes the methods and findings of included studies.

\section{Risk of bias}

Owing to the high degree of heterogeneity, it was difficult to quantitatively compare studies' risk of bias. Drawing on the categories for assessment of risk of bias in the ROBINS-I v.19 tool, ${ }^{40}$ we systematically assessed all 20 included articles for potentially biasing limitations in the domains of confounding, comparison groups, data collection, lack of intervention detail and outcome assessment. We found that common biases to

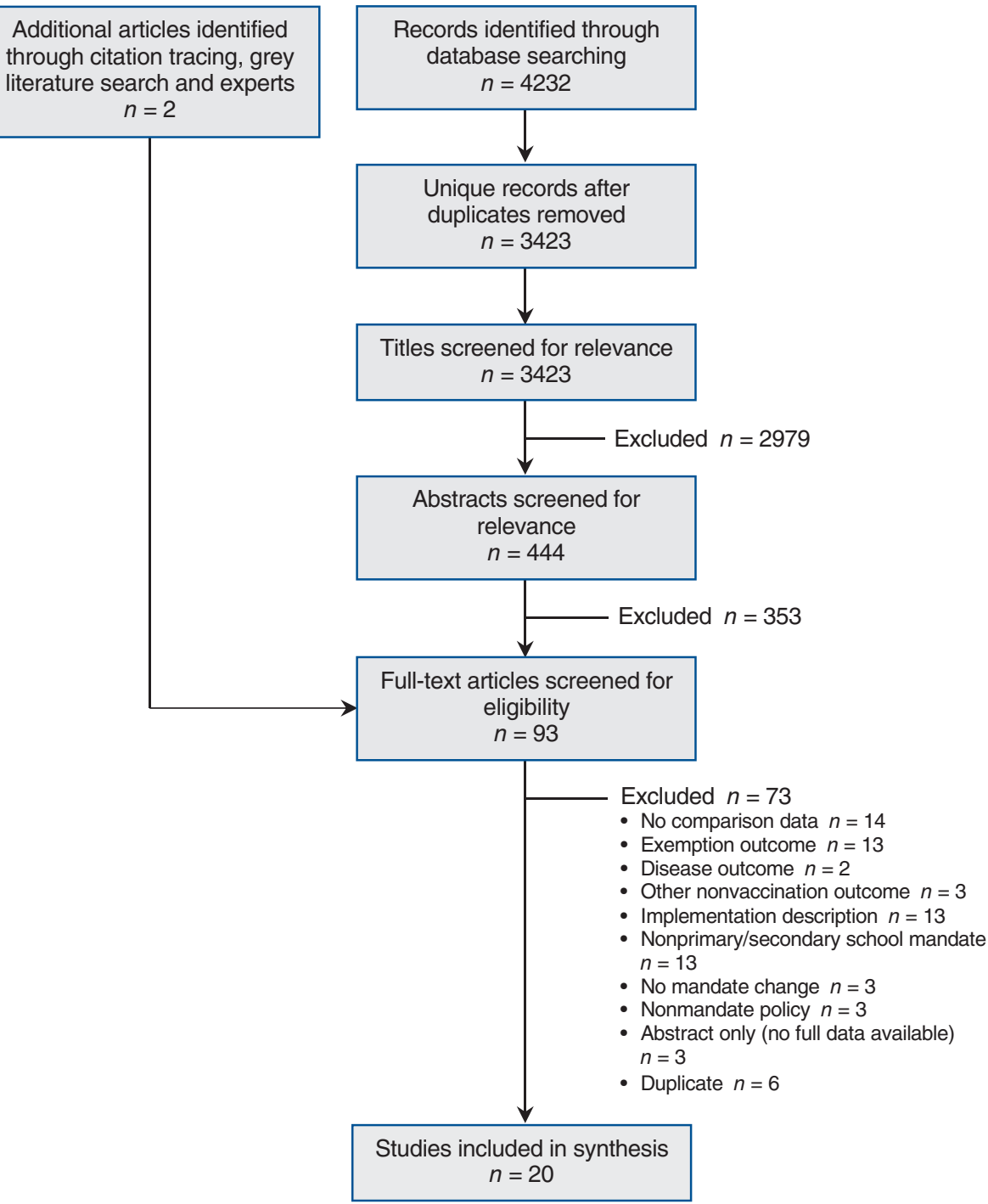

Figure 1: Flow diagram showing study selection. 


\begin{tabular}{|c|c|c|c|c|c|c|}
\hline $\begin{array}{l}\text { Investigator/ } \\
\text { year }\end{array}$ & Setting & Method & $\begin{array}{l}\text { Data source } \\
\text { (population) }\end{array}$ & $\begin{array}{l}\text { Mandate change } \\
\text { studied (year) }\end{array}$ & Outcome of interest & Main findings \\
\hline $\begin{array}{l}\text { Averhoff et } \\
\text { al., }{ }^{23} 2004\end{array}$ & $\begin{array}{l}\text { San Diego, } \\
\text { Calif., US }\end{array}$ & $\begin{array}{l}\text { Uncontrolled } \\
\text { before-after } \\
\text { study using } \\
\text { survey data }\end{array}$ & $\begin{array}{l}\text { Random-digit-dial } \\
\text { telephone survey in } \\
1998(n=205) \text { and } \\
1999(n=378)\end{array}$ & $\begin{array}{l}7 \text { th grade } \\
\text { hepatitis B and } \\
\text { MMR mandate } \\
(1999)\end{array}$ & $\begin{array}{l}\text { 3-dose hepatitis B } \\
\text { and 2-dose MMR }\end{array}$ & $\begin{array}{l}\text { - In year mandate took } \\
\text { effect, } 7 \text { th grade } \\
\text { students more likely to } \\
\text { be vaccinated than other } \\
\text { cohorts not subject to } \\
\text { mandate } \\
\text { - Effect was larger for } \\
\text { hepatitis B, which had } \\
\text { lower uptake before } \\
\text { mandate } \\
\text { - No other factors found to } \\
\text { be significantly } \\
\text { associated with being } \\
\text { vaccinated }\end{array}$ \\
\hline $\begin{array}{l}\text { Carpenter } \\
\text { et al., }{ }^{25} 2019\end{array}$ & US & $\begin{array}{l}\text { Difference-in- } \\
\text { differences } \\
\text { analysis } \\
\text { based on } \\
\text { retrospective } \\
\text { analysis of } \\
\text { data from } \\
\text { cross-sectional } \\
\text { vaccination } \\
\text { coverage } \\
\text { survey }\end{array}$ & $\begin{array}{l}\text { NIS-Teen 2008- } \\
2013 \text { (including } \\
\text { cellphone from } 2011 \\
\text { onward) } \\
(n=116403)\end{array}$ & $\begin{array}{l}\text { Middle school } \\
\text { Tdap vaccination } \\
\text { mandate } \\
(2005-2015)\end{array}$ & $\begin{array}{l}\text { Increase in Tdap } \\
\text { coverage at age } \\
10-13 \text { yr in states } \\
\text { with Tdap } \\
\text { mandates }\end{array}$ & $\begin{array}{l}\text { Tdap uptake about } 13 \% \\
\text { higher in states with } \\
\text { mandates, with spillover } \\
\text { effects to other vaccines } \\
\text { (HPV and MCV) }\end{array}$ \\
\hline $\begin{array}{l}\text { Cuff et al., }{ }^{26} \\
2016\end{array}$ & Virginia, US & $\begin{array}{l}\text { Prospective } \\
\text { cohort study } \\
\text { using } \\
\text { administrative } \\
\text { data and } \\
\text { telephone } \\
\text { survey }\end{array}$ & $\begin{array}{l}\text { University of Virginia } \\
\text { Clinical Data } \\
\text { Repository } 2014 \\
\text { ( } n=908 \text { girls) }\end{array}$ & $\begin{array}{l}\text { 6th grade HPV } \\
\text { mandate for girls } \\
(2009)\end{array}$ & $\begin{array}{l}\text { HPV vaccine } \\
\text { initiation ( } \geq 1 \text { dose) } \\
\text { in girls } 11-12 \text { yr of } \\
\text { age and proportion } \\
\text { vaccinated in } 2009 \\
\text { and } 2014 \text { cohorts }\end{array}$ & $\begin{array}{l}\text { Mandate had no effect on } \\
\text { HPV coverage } 5 \text { yr after } \\
\text { mandate implementation }\end{array}$ \\
\hline
\end{tabular}




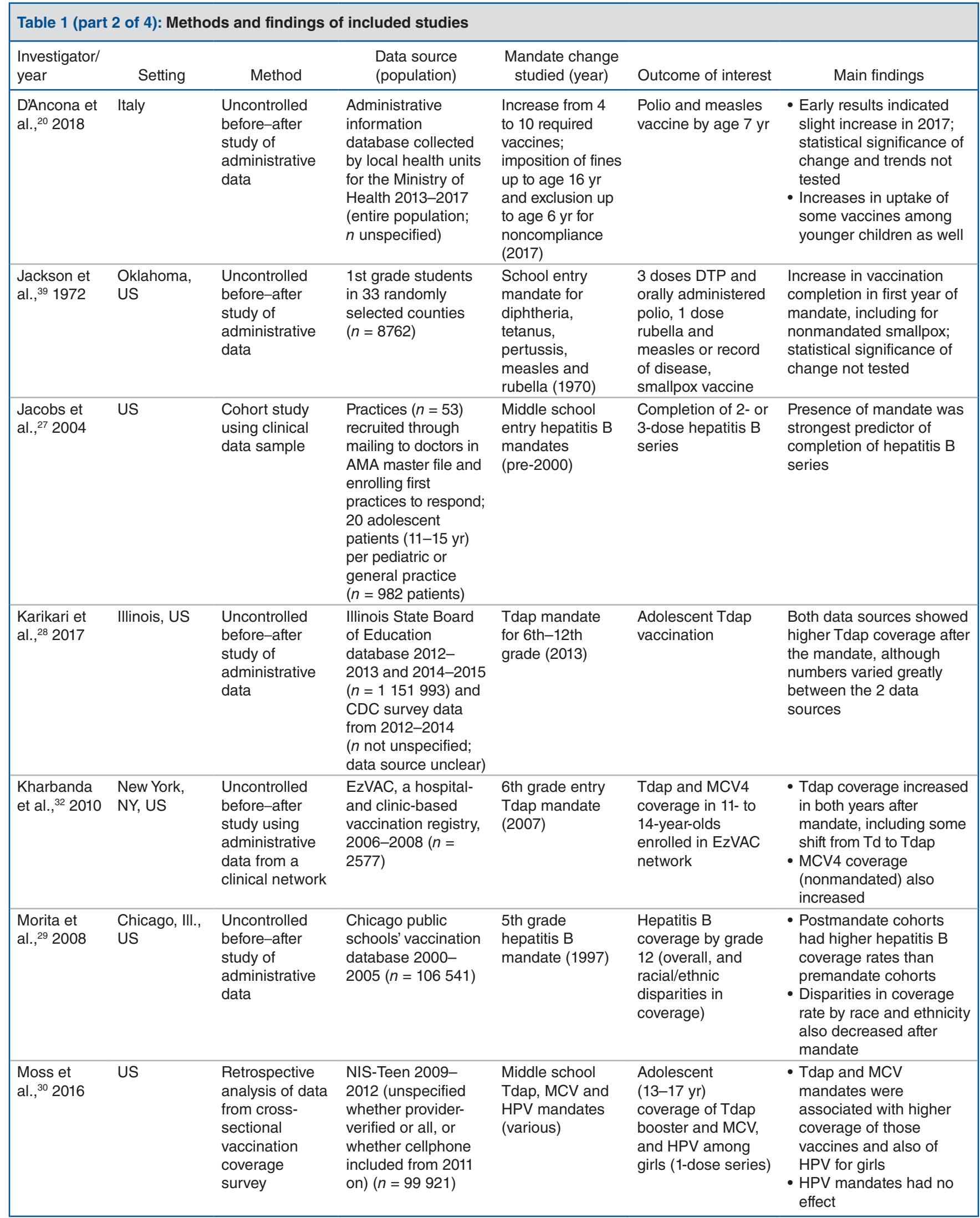




\begin{tabular}{|c|c|c|c|c|c|c|}
\hline $\begin{array}{l}\text { Investigator/ } \\
\text { year }\end{array}$ & Setting & Method & $\begin{array}{l}\text { Data source } \\
\text { (population) }\end{array}$ & $\begin{array}{l}\text { Mandate change } \\
\text { studied (year) }\end{array}$ & Outcome of interest & Main findings \\
\hline $\begin{array}{l}\text { Olshen et } \\
\text { al., }{ }^{31} 2007\end{array}$ & $\begin{array}{l}27 \text { US states } \\
+D C\end{array}$ & $\begin{array}{l}\text { Cross-sectional } \\
\text { study }\end{array}$ & $\begin{array}{l}\text { Health Plan } \\
\text { Employer Data and } \\
\text { Information Set } \\
2003(n=100000)\end{array}$ & $\begin{array}{l}\text { Mandates for } \\
\text { hepatitis B and } \\
\text { varicella before } \\
2003 \text { (various) }\end{array}$ & $\begin{array}{l}\text { Policy attribute that } \\
\text { is associated with } \\
\text { higher mean } \\
\text { coverage }\end{array}$ & $\begin{array}{l}\text { - Mandate policy at middle } \\
\text { school level was } \\
\text { associated with higher } \\
\text { mean hepatitis B and } \\
\text { varicella coverage } \\
\text { - Other policy attributes } \\
\text { (e.g., exemptions, } \\
\text { payment and } \\
\text { deductibles, universal } \\
\text { purchasing) not } \\
\text { associated }\end{array}$ \\
\hline $\begin{array}{l}\text { Perkins et } \\
\text { al., }{ }^{33} 2016\end{array}$ & US & $\begin{array}{l}\text { Retrospective } \\
\text { analysis of data } \\
\text { from cross- } \\
\text { sectional } \\
\text { vaccination } \\
\text { coverage } \\
\text { survey }\end{array}$ & $\begin{array}{l}\text { NIS-Teen 2009- } \\
2013 \text { (provider- } \\
\text { verified responses } \\
\text { only; unspecified } \\
\text { whether cellphone } \\
\text { included from } 2011 \\
\text { on) ( } n=47845 \\
\text { parents of girls) }\end{array}$ & $\begin{array}{l}\text { Middle school } \\
\text { HPV mandate for } \\
\text { girls (DC, } \\
\text { Virginia) and } \\
\text { HPV education } \\
\text { mandate } \\
\text { (Louisiana, } \\
\text { Michigan, } \\
\text { Colorado, } \\
\text { Indiana, lowa, } \\
\text { Illinois, New } \\
\text { Jersey, North } \\
\text { Carolina, Texas, } \\
\text { Washington) } \\
\text { (various) }\end{array}$ & $\begin{array}{l}\text { HPV vaccine } \\
\text { coverage (series } \\
\text { initiation, } \\
\text { completion) in girls }\end{array}$ & $\begin{array}{l}\text { No difference in HPV } \\
\text { coverage between girls in } \\
\text { states with school entry } \\
\text { vaccine mandates or } \\
\text { education mandates } \\
\text { compared to no mandates }\end{array}$ \\
\hline $\begin{array}{l}\text { Potter et } \\
\text { al., }{ }^{35} 2014\end{array}$ & $\begin{array}{l}\text { Michigan, } \\
\text { US }\end{array}$ & $\begin{array}{l}\text { Uncontrolled } \\
\text { before-after } \\
\text { study using } \\
\text { administrative } \\
\text { data }\end{array}$ & $\begin{array}{l}\text { Michigan Care } \\
\text { Improvement } \\
\text { Registry (statewide } \\
\text { vaccination registry) } \\
2009 \text { and } 2010 \\
(2009 n=133738 \\
2010 n=131051)\end{array}$ & $\begin{array}{l}\text { New mandate at } \\
6 \text { th grade entry } \\
\text { for Tdap, MCV4, } \\
\text { varicella (2010) }\end{array}$ & $\begin{array}{l}\text { Completion of all } \\
\text { required vaccines } \\
\text { (as a single } \\
\text { variable); time to } \\
\text { completion } \\
\text { (up-to-date status) } \\
\text { of all required } \\
\text { vaccines; initiation } \\
\text { of HPV vaccine } \\
\text { (girls only) }\end{array}$ & $\begin{array}{l}\text { Vaccine completion (up to } \\
\text { date for all) was higher in } \\
\text { year after mandate, and } \\
\text { time to completion was } \\
\text { shorter }\end{array}$ \\
\hline $\begin{array}{l}\text { Simpson et } \\
\text { al., }{ }^{36} 2013\end{array}$ & Arizona, US & $\begin{array}{l}\text { Uncontrolled } \\
\text { before-after } \\
\text { study using } \\
\text { administrative } \\
\text { data }\end{array}$ & $\begin{array}{l}\text { Arizona State } \\
\text { Immunization } \\
\text { Information System } \\
2006-2011(n= \\
954953 \text { records) }\end{array}$ & $\begin{array}{l}\text { New mandate for } \\
\text { MCV4 for 6th } \\
\text { grade entry if } \\
\text { aged } \geq 11 \mathrm{yr} \\
(2008)\end{array}$ & MCV4 coverage & $\begin{array}{l}\text { Vaccine coverage for } \\
12 \text {-year-olds was higher } \\
\text { after mandate than before } \\
\text { mandate }\end{array}$ \\
\hline
\end{tabular}




\begin{tabular}{|c|c|c|c|c|c|c|}
\hline $\begin{array}{l}\text { Investigator/ } \\
\text { year }\end{array}$ & Setting & Method & $\begin{array}{l}\text { Data source } \\
\text { (population) }\end{array}$ & $\begin{array}{l}\text { Mandate change } \\
\text { studied (year) }\end{array}$ & Outcome of interest & Main findings \\
\hline $\begin{array}{l}\text { Thompson } \\
\text { et al.,21 } \\
1994\end{array}$ & $\begin{array}{l}\text { Victoria, } \\
\text { Australia }\end{array}$ & $\begin{array}{l}\text { Uncontrolled } \\
\text { before-after } \\
\text { study using } \\
\text { administrative } \\
\text { data }\end{array}$ & $\begin{array}{l}\text { Victoria Directorate } \\
\text { of School Education } \\
\text { mid-year census } \\
1991 \text { and } 1992 \\
\text { (1576 schools } \\
\text { included; } 1992 n= \\
45049 \text { students) }\end{array}$ & $\begin{array}{l}\text { Documentation } \\
\text { mandate for } \\
\text { school entry } \\
(1992)\end{array}$ & $\begin{array}{l}\text { Submitted } \\
\text { documentation of } \\
\text { immunization } \\
\text { status; } \\
\text { documentation of } \\
\text { complete (up-to- } \\
\text { date) vaccination } \\
\text { for age }\end{array}$ & $\begin{array}{l}\text { Small increase in } \\
\text { submitted documentation } \\
\text { after policy mandate, } \\
\text { including small increase in } \\
\text { documentation of fully } \\
\text { vaccinated students and } \\
\text { larger increase in } \\
\text { documentation of } \\
\text { incompletely vaccinated } \\
\text { students }\end{array}$ \\
\hline $\begin{array}{l}\text { Thompson } \\
\text { et al., }{ }^{37} 2018\end{array}$ & $\begin{array}{l}\text { Rhode } \\
\text { Island, US }\end{array}$ & $\begin{array}{l}\text { Retrospective } \\
\text { analysis of data } \\
\text { from cross- } \\
\text { sectional } \\
\text { vaccination } \\
\text { coverage } \\
\text { survey }\end{array}$ & $\begin{array}{l}\text { NIS-Teen 2010- } \\
2016 \text { (unspecified } \\
\text { whether cellphone } \\
\text { included from } 2011 \\
\text { on; parent report } \\
\text { only) ( } n \text { unspecified) }\end{array}$ & $\begin{array}{l}\text { HPV mandate for } \\
\text { initiation by } 7 \text { th } \\
\text { grade and } \\
\text { completion by } 9 \text { th } \\
\text { grade (2015) }\end{array}$ & $\begin{array}{l}\text { Initiation of HPV } \\
\text { series }\end{array}$ & $\begin{array}{l}\text { - Only initiation in boys } \\
\text { showed small increase } \\
\text { after mandate; no } \\
\text { change among girls } \\
\text { - No increase among boys } \\
\text { in other states }\end{array}$ \\
\hline $\begin{array}{l}\text { Wilson et } \\
\text { al., }{ }^{38} 2005\end{array}$ & $\begin{array}{l}\text { Kansas City, } \\
\text { Mo., and } \\
\text { Kansas City, } \\
\text { Kan., US }\end{array}$ & $\begin{array}{l}\text { Retrospective } \\
\text { cohort study of } \\
\text { school samples }\end{array}$ & $\begin{array}{l}\text { Random sample of } \\
\text { vaccine records } \\
\text { from purposive } \\
\text { sample of } 11 \text { high } \\
\text { schools in } 2003 \text { ( } n= \\
2230)\end{array}$ & $\begin{array}{l}\text { Hepatitis B } \\
\text { mandate for } \\
\text { elementary } \\
\text { school (1997) } \\
\text { and middle } \\
\text { school (1999) } \\
\text { (Missouri) }\end{array}$ & $\begin{array}{l}3 \text { hepatitis } B \\
\text { vaccine doses at } \\
\text { 9th grade }\end{array}$ & $\begin{array}{l}\text { - Students affected by } \\
\text { middle school hepatitis B } \\
\text { mandate were more } \\
\text { likely to have been } \\
\text { vaccinated than earlier } \\
\text { cohort in same area or } \\
\text { contemporaries in } \\
\text { comparison area without } \\
\text { mandate } \\
\text { - No spillover differences } \\
\text { observed for MMR or Td } \\
\text { vaccines }\end{array}$ \\
\hline
\end{tabular}

which this body of literature is vulnerable include confounding, selection bias, measurement bias and bias due to deviation from/variation in implementation of the interventions. All of the included studies were observational assessments of natural experiments rather than studies of interventions designed to be implemented in a controlled manner. Fifteen studies used ecological designs, which are prone to confounding and bias, including ecological fallacy, in which associations identified at a group level are extrapolated to apply to individuals ${ }^{41}$ and cannot be relied on as evidence of causality, ${ }^{42}$ as an increase or decrease in individual coverage may be due to factors other than the mandate. Several of the nonecological studies, however, recruited study populations that were unlikely to be representative of the larger population, which limited the external validity (generalizability) of the findings. Although some studies described cointerventions, others made no mention of common cointerventions (e.g., outreach programs to improve vaccination awareness, education and access, or changes in vaccine purchasing, coverage and distribution) that may accompany mandatory policies, and little effort was made across all studies to measure or account for the impact of such potential confounders. Studies with pre-post designs varied greatly in the length of baseline and follow-up data, and some had such short periods that a trend could not reliably be established. Any National Immunization Survey-Teen sample is vulnerable to response bias owing to the moderate response rate $(55.5 \%$ for the landline sample and $29.5 \%$ for the cellphone sample in 2016). ${ }^{43}$ Landline-only samples (including all pre-2011 surveys) are vulnerable to additional selection bias, non-provider-verified data (including nearly half of National Immunization Survey-Teen responses) are vulnerable to recall bias and social desirability bias by the respondent, and using only provider-verified data risks additional selection bias. Finally, although implementation elements such as messaging parents and consistency of enforcement likely matter greatly in a policy's success, details or measures of implementation factors were rarely mentioned in the studies and were never accounted for in analyses. Table 2 presents this assessment for each study individually, and discussion of these potentially biasing limitations is integrated into the findings below.

\section{Study findings}

School entry mandates (typically for age 5-7 yr and applying to a large array of vaccines scheduled from birth to school entry) were found to be associated with increased documentation and/or vaccination in diverse settings. The earliest study 


\begin{tabular}{|c|c|c|c|c|c|}
\hline Investigator & $\begin{array}{l}\text { Confounders, including } \\
\text { cointerventions } \\
\text { (ecological fallacy, } \\
\text { confounding) }\end{array}$ & $\begin{array}{l}\text { Bias in comparison } \\
\text { groups (selection } \\
\text { bias) }\end{array}$ & $\begin{array}{l}\text { Data collection issues and } \\
\text { missing data (selection } \\
\text { bias, nonresponse bias, } \\
\text { information biases } \\
\text { including recall bias and } \\
\text { reporting bias) }\end{array}$ & $\begin{array}{l}\text { Lack of detail } \\
\text { regarding intervention } \\
\text { or implementation } \\
\text { (bias due to deviation } \\
\text { from or variation in } \\
\text { interventions) }\end{array}$ & $\begin{array}{c}\text { Outcome } \\
\text { assessment } \\
\text { methods or } \\
\text { measures } \\
\text { (measurement bias) }\end{array}$ \\
\hline $\begin{array}{l}\text { Averhoff et } \\
\text { al. }{ }^{23}\end{array}$ & $\begin{array}{l}\text { No measurement or } \\
\text { adjustment for } \\
\text { important potential } \\
\text { confounders (e.g., } \\
\text { home learning rates, } \\
\text { noncompliance) }\end{array}$ & - & $\begin{array}{l}\text { Self-reported vaccination } \\
\text { data from single school } \\
\text { district; response rate } \\
\text { unknown }\end{array}$ & $\begin{array}{l}\text { Exemption process, } \\
\text { consequences for } \\
\text { noncompliance } \\
\text { and other } \\
\text { implementation } \\
\text { factors not specified }\end{array}$ & $\begin{array}{l}\text { No external } \\
\text { verification of } \\
\text { vaccination status }\end{array}$ \\
\hline $\begin{array}{l}\text { Bugenske et } \\
\text { al. }{ }^{24}\end{array}$ & $\begin{array}{l}\text { - Ecological study } \\
\text { - No measurement or } \\
\text { adjustment for } \\
\text { important potential } \\
\text { confounders }\end{array}$ & $\begin{array}{l}\text { May have been } \\
\text { unobserved } \\
\text { differences in } \\
\text { individuals between } \\
\text { states with and } \\
\text { without mandates }\end{array}$ & $\begin{array}{l}\text { Analysis limited to landline } \\
\text { telephones and responses } \\
\text { accompanied by provider- } \\
\text { verified records; may not } \\
\text { be representative }\end{array}$ & $\begin{array}{l}\text { Policies were } \\
\text { grouped together, not } \\
\text { allowing for analysis } \\
\text { of subtle differences } \\
\text { in implementation or } \\
\text { context }\end{array}$ & $\begin{array}{l}\text { Follow-up time for } \\
\text { policies limited; } \\
\text { up-to-date } \\
\text { vaccination status } \\
\text { defined as } 1 \text { dose }\end{array}$ \\
\hline $\begin{array}{l}\text { Carpenter et } \\
\text { al. }{ }^{25}\end{array}$ & Ecological study & $\begin{array}{l}\text { - May have been } \\
\text { unobserved } \\
\text { differences in } \\
\text { individuals } \\
\text { between states } \\
\text { with and without } \\
\text { mandates } \\
\text { - Age groups as } \\
\text { proxy for middle } \\
\text { school enrolment } \\
\text { may not reflect } \\
\text { actual grades } \\
\text { affected by } \\
\text { mandates in every } \\
\text { state }\end{array}$ & $\begin{array}{l}\text { Used } 2008 \text { data as proxy } \\
\text { for premandate } 2004 / 05 \\
\text { vaccination status; no } \\
\text { middle school enrolment } \\
\text { data; no premandate data }\end{array}$ & $\begin{array}{l}\text { Multiple state policies } \\
\text { grouped together; no } \\
\text { accounting for } \\
\text { differences }\end{array}$ & - \\
\hline Cuff et al. ${ }^{26}$ & $\begin{array}{l}\text { No measurement or } \\
\text { adjustment for important } \\
\text { potential confounders }\end{array}$ & - & $\begin{array}{l}\text { Single-centre study; low } \\
\text { response rate; participants } \\
\text { included only parents } \\
\text { seeking care for well-child } \\
\text { care visits; may not be } \\
\text { representative }\end{array}$ & - & $\begin{array}{l}\text { Only } 1 \mathrm{yr} \text { of baseline } \\
\text { (premandate) data }\end{array}$ \\
\hline $\begin{array}{l}\text { D'Ancona et } \\
\text { al. }^{20}\end{array}$ & $\begin{array}{l}\text { - Ecological study } \\
\text { - Media campaign } \\
\text { cointervention not } \\
\text { accounted for }\end{array}$ & - & - & - & $\begin{array}{l}\text { Lack of reliable } \\
\text { denominator; no } \\
\text { testing for statistical } \\
\text { significance of } \\
\text { changes; only } 1 \mathrm{yr} \text { of } \\
\text { postmandate data }\end{array}$ \\
\hline $\begin{array}{l}\text { Jackson et } \\
\text { al. } .^{39}\end{array}$ & $\begin{array}{l}\text { - Ecological study } \\
\text { - Known cointerventions } \\
\text { included awareness } \\
\text { campaigns to public } \\
\text { and doctors, improving } \\
\text { access through } \\
\text { increased clinic days } \\
\text { and free vaccines, } \\
\text { special measles } \\
\text { vaccination campaign } \\
\text { in preintervention year, } \\
\text { rubella vaccine } \\
\text { shortage in } \\
\text { preintervention year; } \\
\text { effects not measured } \\
\text { separately }\end{array}$ & - & $\begin{array}{l}\text { Convenience sample of } \\
\text { schoolchildren (not } \\
\text { random) from a random } \\
\text { sample of school districts }\end{array}$ & - & $\begin{array}{l}\text { No external } \\
\text { verification of } \\
\text { vaccination status } \\
\text { (parent report); no } \\
\text { testing for statistical } \\
\text { significance of } \\
\text { changes; only } 1 \text { yr of } \\
\text { pre- and } \\
\text { postmandate data }\end{array}$ \\
\hline
\end{tabular}




\begin{tabular}{|c|c|c|c|c|c|}
\hline Investigator & $\begin{array}{l}\text { Confounders, including } \\
\text { cointerventions } \\
\text { (ecological fallacy, } \\
\text { confounding) }\end{array}$ & $\begin{array}{c}\text { Bias in comparison } \\
\text { groups (selection } \\
\text { bias) }\end{array}$ & $\begin{array}{l}\text { Data collection issues and } \\
\text { missing data (selection } \\
\text { bias, nonresponse bias, } \\
\text { information biases } \\
\text { including recall bias and } \\
\text { reporting bias) }\end{array}$ & $\begin{array}{l}\text { Lack of detail } \\
\text { regarding intervention } \\
\text { or implementation } \\
\text { (bias due to deviation } \\
\text { from or variation in } \\
\text { interventions) }\end{array}$ & $\begin{array}{c}\text { Outcome } \\
\text { assessment } \\
\text { methods or } \\
\text { measures } \\
\text { (measurement bias) }\end{array}$ \\
\hline $\begin{array}{l}\text { Jacobs et } \\
\text { al. }{ }^{27}\end{array}$ & Ecological study & $\begin{array}{l}\text { May have been } \\
\text { unobserved } \\
\text { differences in } \\
\text { individuals between } \\
\text { states with and } \\
\text { without mandates }\end{array}$ & $\begin{array}{l}\text { - Convenience sample of } \\
\text { pediatric and family } \\
\text { practices; adolescents } \\
\text { enrolled only after visiting } \\
\text { doctor; } 7 \% \text { excluded } \\
\text { owing to incomplete } \\
\text { records; unclear how } \\
\text { representative this clinical } \\
\text { sample is of population } \\
\text { - Lack of clarity regarding } \\
\text { data collection timelines }\end{array}$ & $\begin{array}{l}\text { Policies grouped } \\
\text { together, not allowing } \\
\text { for analysis of subtle } \\
\text { differences in } \\
\text { implementation or } \\
\text { context }\end{array}$ & - \\
\hline $\begin{array}{l}\text { Karikari et } \\
\text { al. }{ }^{28}\end{array}$ & $\begin{array}{l}\text { - Ecological study } \\
\text { - No measurement or } \\
\text { adjustment for } \\
\text { important potential } \\
\text { confounders }\end{array}$ & - & $\begin{array}{l}\text { Included only school- } \\
\text { enrolled children in } \\
\text { vaccination registry; } \\
2 \text { different data sources for } \\
\text { outcome had different } \\
\text { results; unclear why } \\
\text { differences existed; lack of } \\
\text { detail on CDC survey }\end{array}$ & $\begin{array}{l}\text { No information on } \\
\text { implementation or } \\
\text { context }\end{array}$ & $\begin{array}{l}\text { Unknown to what } \\
\text { extent findings can } \\
\text { be extrapolated to } \\
\text { larger population }\end{array}$ \\
\hline $\begin{array}{l}\text { Kharbanda } \\
\text { et al. }{ }^{32}\end{array}$ & $\begin{array}{l}\text { No measurement or } \\
\text { adjustment for potential } \\
\text { confounders, although } \\
\text { did look at spillover } \\
\text { effect on nonmandate } \\
\text { vaccination }\end{array}$ & - & $\begin{array}{l}\text { - Population from system } \\
\text { of only } 1 \text { hospital; not } \\
\text { representative of larger } \\
\text { population; may not be } \\
\text { generalizable } \\
\text { - Data missing on any } \\
\text { vaccines given outside } \\
\text { participating hospital } \\
\text { system } \\
\text { - Only included those with } \\
\text { sufficient vaccination } \\
\text { information }\end{array}$ & $\begin{array}{l}\text { No information on } \\
\text { implementation or } \\
\text { context }\end{array}$ & - \\
\hline $\begin{array}{l}\text { Morita et } \\
\text { al. }{ }^{29}\end{array}$ & Ecological study & - & $\begin{array}{l}\text { Losses to follow-up } \\
\text { (e.g., students leaving } \\
\text { school) excluded from } \\
\text { analysis }\end{array}$ & $\begin{array}{l}\text { Likely inconsistent } \\
\text { enforcement of policy, } \\
\text { not captured by study } \\
\text { data collection } \\
\text { methods }\end{array}$ & $\begin{array}{l}\text { Only } 2 \mathrm{yr} \text { of } \\
\text { postmandate data }\end{array}$ \\
\hline Moss et al. ${ }^{30}$ & $\begin{array}{l}\text { - Ecological study } \\
\text { - No measurement or } \\
\text { adjustment for } \\
\text { important potential } \\
\text { confounders }\end{array}$ & $\begin{array}{l}\text { May have been } \\
\text { unobserved } \\
\text { differences in setting } \\
\text { between states with } \\
\text { and without } \\
\text { mandates }\end{array}$ & - & $\begin{array}{l}\text { Likely inconsistent } \\
\text { enforcement of policy, } \\
\text { not captured by study } \\
\text { data collection } \\
\text { methods }\end{array}$ & $\begin{array}{l}\text { Unspecified/ } \\
\text { unadjusted for state } \\
\text { differences in age/ } \\
\text { grade of mandate }\end{array}$ \\
\hline $\begin{array}{l}\text { Olshen et } \\
\text { al. }{ }^{31}\end{array}$ & Ecological study & $\begin{array}{l}\text { May have been } \\
\text { differences in } \\
\text { population with study } \\
\text { insurer and } \\
\text { population as a } \\
\text { whole } \\
\text { (representativeness } \\
\text { and generalizability) }\end{array}$ & - & $\begin{array}{l}\text { Policies grouped } \\
\text { together, not allowing } \\
\text { for subtle differences } \\
\text { in implementation or } \\
\text { context }\end{array}$ & $\begin{array}{l}\text { Full model } \\
\text { information not } \\
\text { provided }\end{array}$ \\
\hline Omer et al. ${ }^{22}$ & $\begin{array}{l}\text { Other known changes } \\
\text { (e.g., in vaccination } \\
\text { schedule, exemption } \\
\text { forms) before policy } \\
\text { change appear to have } \\
\text { affected trends }\end{array}$ & - & $\begin{array}{l}\text { Home learners may not } \\
\text { have been included }\end{array}$ & - & - \\
\hline
\end{tabular}




\begin{tabular}{|c|c|c|c|c|c|}
\hline Investigator & $\begin{array}{l}\text { Confounders, including } \\
\text { cointerventions } \\
\text { (ecological fallacy, } \\
\text { confounding) }\end{array}$ & $\begin{array}{c}\text { Bias in comparison } \\
\text { groups (selection } \\
\text { bias) }\end{array}$ & $\begin{array}{l}\text { Data collection issues and } \\
\text { missing data (selection } \\
\text { bias, nonresponse bias, } \\
\text { information biases } \\
\text { including recall bias and } \\
\text { reporting bias) }\end{array}$ & $\begin{array}{l}\text { Lack of detail } \\
\text { regarding intervention } \\
\text { or implementation } \\
\text { (bias due to deviation } \\
\text { from or variation in } \\
\text { interventions) }\end{array}$ & $\begin{array}{c}\text { Outcome } \\
\text { assessment } \\
\text { methods or } \\
\text { measures } \\
\text { (measurement bias) }\end{array}$ \\
\hline $\begin{array}{l}\text { Perkins et } \\
\text { al. }^{33}\end{array}$ & Ecological study & $\begin{array}{l}\text { May have been } \\
\text { unobserved } \\
\text { differences in setting } \\
\text { between states with } \\
\text { and without mandates }\end{array}$ & $\begin{array}{l}\text { Included only respondents } \\
\text { with adequate provider- } \\
\text { verified vaccination history }\end{array}$ & $\begin{array}{l}\text { Policies grouped } \\
\text { together, not allowing } \\
\text { for subtle differences } \\
\text { in implementation or } \\
\text { context }\end{array}$ & $\begin{array}{l}\text { Only } 1 \text { yr of baseline } \\
\text { (premandate) data }\end{array}$ \\
\hline $\begin{array}{l}\text { Pierre-Victor } \\
\text { et al. }{ }^{34}\end{array}$ & Ecological study & $\begin{array}{l}\text { May have been } \\
\text { unobserved } \\
\text { differences in setting } \\
\text { between states with } \\
\text { and without mandates }\end{array}$ & $\begin{array}{l}\text { Landline-only sample; } \\
\text { analysis included only } \\
\text { those who responded } \\
\text { about HPV }\end{array}$ & - & - \\
\hline Potter et al. ${ }^{35}$ & Ecological study & - & $\begin{array}{l}\text { Home learners may not } \\
\text { have been included }\end{array}$ & - & $\begin{array}{l}\text { Only } 1 \text { yr of baseline } \\
\text { (premandate) and } \\
\text { follow-up } \\
\text { (postmandate) data }\end{array}$ \\
\hline $\begin{array}{l}\text { Simpson et } \\
\text { al. }{ }^{36}\end{array}$ & $\begin{array}{l}\text { - Ecological study } \\
\text { - No measurement or } \\
\text { adjustment for } \\
\text { important potential } \\
\text { confounders; known } \\
\text { potential confounders } \\
\text { include } 2005 \mathrm{ACIP} \\
\text { recommendation and } \\
\text { education/awareness } \\
\text { campaign that } \\
\text { accompanied mandate }\end{array}$ & - & $\begin{array}{l}\text { Comparison with census } \\
\text { data indicates registry may } \\
\text { have underestimated } \\
\text { coverage }\end{array}$ & - & - \\
\hline $\begin{array}{l}\text { Thompson } \\
\text { et al. }{ }^{21}\end{array}$ & Ecological study & - & $\begin{array}{l}\text { Data not available from } \\
\text { nongovernmental schools; } \\
\text { only schools with } \\
\text { kindergarten enrolment } \\
\text { included }\end{array}$ & $\begin{array}{l}\text { Not possible to know } \\
\text { reason for missing } \\
\text { documentation, so } \\
\text { unclear whether this } \\
\text { represents bias in } \\
\text { coverage outcome; } \\
\text { some schools may } \\
\text { have been more } \\
\text { compliant than others }\end{array}$ & $\begin{array}{l}\text { Limited pre- and } \\
\text { postmandate data }\end{array}$ \\
\hline $\begin{array}{l}\text { Thompson } \\
\text { et al. }{ }^{37}\end{array}$ & $\begin{array}{l}\text { - Ecological study } \\
\text { - No measurement or } \\
\text { control for potential } \\
\text { confounders } \\
\text { - Insurance coverage } \\
\text { for HPV for boys in } \\
\text { other states unknown } \\
\text { and may have } \\
\text { confounded uptake }\end{array}$ & $\begin{array}{l}\text { "All other states" } \\
\text { comparator includes } \\
\text { states both with and } \\
\text { without mandates }\end{array}$ & $\begin{array}{l}\text { Parent report only (no } \\
\text { provider verification) }\end{array}$ & $\begin{array}{l}\text { Implementation } \\
\text { details not specified } \\
\text { other than difficult to } \\
\text { opt out }\end{array}$ & $\begin{array}{l}\text { Only } 1 \mathrm{yr} \text { of } \\
\text { postmandate data }\end{array}$ \\
\hline $\begin{array}{l}\text { Wilson et } \\
\text { al. }{ }^{38}\end{array}$ & $\begin{array}{l}\text { Many cointerventions } \\
\text { described; no } \\
\text { measurement or control } \\
\text { for potential confounders }\end{array}$ & $\begin{array}{l}\text { Small school-based } \\
\text { population may not } \\
\text { be representative; } \\
\text { combination of } \\
\text { random and } \\
\text { purposive sampling; } \\
1 \text { school excluded } \\
\text { owing to improper } \\
\text { documentation; } \\
\text { nonenrolled students } \\
\text { excluded (potential } \\
\text { selection bias; } \\
\text { enrolment in rural } \\
\text { areas below target }\end{array}$ & - & $\begin{array}{l}\text { Implementation } \\
\text { details not specified }\end{array}$ & $\begin{array}{l}\text { Small sample, } \\
\text { insufficient statistical } \\
\text { power }\end{array}$ \\
\hline
\end{tabular}


showed increased coverage for all vaccines, including one not required by the mandate, in the first year of a 1970 vaccine mandate compared to the previous year. ${ }^{39}$ Thompson and colleagues' ${ }^{21}$ evaluation of an Australian mandate for school entry vaccination certificates in the 1990s showed increased documentation for all students, with greatest effect among those not up to date with vaccines. Omer and colleagues ${ }^{22}$ studied changes to a Washington State vaccine mandate that introduced a requirement for a health care provider signature for exemptions in 2011 and found an increase in the proportion of students who were up to date for all vaccines after implementation. D'Ancona and colleagues ${ }^{20}$ reported preliminary numbers following Italy's 2017 decision to add vaccination to an existing mandate and enforce it. They found a small increase in measles/mumps/rubella and polio vaccination among 7-year-old children. Jackson and Carpenter, ${ }^{39}$ Thompson and colleagues ${ }^{21}$ and D'Ancona and colleagues ${ }^{20}$ did not assess the statistical significance of the observed changes. All 3 studies were ecological studies and, thus, vulnerable to confounding, and the investigators reported varying numbers of cointerventions such as awareness campaigns, and reduction of cost and access barriers. The mandate change studied by Omer and colleagues ${ }^{22}$ was accompanied by other changes in vaccination education and access, which were not controlled for or assessed. Although the evidence for causality in this group of studies was not strong, all associations were positive.

Twelve of the included studies focused primarily or entirely on adolescent mandates (commonly for grades 5-9, or middle school populations) for vaccines other than human papillomavirus (HPV). These vaccines included hepatitis B, tetanus/diphtheria/acellular pertussis, meningococcal vaccines, measles/mumps/rubella and varicella. All 12 studies were conducted in the US, in jurisdictions with preexisting school entry mandates. These studies showed no increase in uptake associated with educational mandates, ${ }^{24}$ but vaccination mandates for these vaccines were positively associated with higher coverage after implementation, ${ }^{23-25,27-30,32,35,36,38}$ regardless of data source or study design. Effect sizes varied greatly; in some studies, larger increases were associated with mandates for vaccines whose coverage was lower before the intervention ${ }^{23,32}$ or for low-income students, ${ }^{25}$ and in 1 study, racial/ethnic disparities in coverage were smaller after the intervention. ${ }^{29}$ Spillover effects were observed from 1 mandated vaccine (e.g., tetanus/diphtheria/acellular pertussis) onto other adolescent vaccines (e.g., meningococcal vaccines) in 3 of the 4 studies in which this was examined. ${ }^{25,30,32}$ The 1 study that showed no spillover effects from tetanus/diphtheria/acellular pertussis mandates involved a small school-based sample that was unlikely to be representative of the entire population. ${ }^{38}$

The evidence on adolescent HPV mandates in the US told a different story. Four studies did not show an association between HPV vaccine education or mandates for girls and an increase in HPV vaccine coverage. . $^{26,33,34}$ Pierre-Victor and colleagues $^{34}$ did find that, independent of mandates, physician recommendation and health care contacts were predictors of HPV vaccination. Thompson and colleagues ${ }^{37}$ studied a later-implemented mandate for both girls and boys in a state with high coverage in girls before the mandate and found that rates increased among boys but not girls following the mandate. The study did not disentangle the effect of the mandate and the expanded insurance coverage for boys, which happened at the same time. Three studies showed a small spillover effect of new adolescent mandates for other vaccines onto HPV vaccine uptake, ${ }^{25,30,35}$ but only if $H P V$ vaccination was not mandated. ${ }^{30}$ This spillover effect was most pronounced among low-income students. ${ }^{25}$ The HPV mandate literature consisted of ecological studies and did not examine or control for other contextual or implementation factors, with the exception of the study by Cuff and colleagues, ${ }^{26}$ which was a single-centre study of well-child clinic patients and may not be representative of the larger population.

\section{Interpretation}

New or tightened requirements for vaccination of schoolchildren were usually associated with increased coverage of the affected populations, with effects appearing larger when preintervention vaccination rates were low. Mandates for HPV vaccination in the US, where there was a high degree of population hesitancy and politicization around the vaccine, were notably ineffective. Spillover effects indicate that health care interactions may be more important than the compulsory nature of mandates and notably had greater impact on HPV vaccine uptake than direct mandates for education or vaccination. The vast majority of the studies were ecological and, thus, vulnerable to confounding and ecological fallacy. Although ecological analyses are important for generating hypotheses and are widely used in epidemiology, they are vulnerable to identifying associations between factors that may be correlative, bidirectional, mediated by other factors, or confounded by unobserved cointerventions or population attributes. Such studies are therefore not typically considered capable of drawing causal conclusions. Few studies examined or accounted for the influence of common cointerventions such as improved access, education, insurance coverage, and changes in vaccine purchasing or costs. Furthermore, implementation and enforcement of vaccine mandates were not examined in most studies, yet poor implementation and/or uneven enforcement may render a policy ineffective. ${ }^{44}$

MacDonald and colleagues ${ }^{45}$ outlined 3 reasons jurisdictions implement vaccination mandates: failure of less-coercive methods of encouraging vaccination, outbreak of vaccinepreventable disease and as a final stage in a global disease-eradication project. Mandates considered in the current analysis whether for documentation, education or vaccination - were enacted in 1 or both of the first 2 of these scenarios, when policy-makers decided such laws were ethically permissible given the safety profile of vaccine supplies and general population acceptance of vaccination. ${ }^{46}$ Although school vaccine mandates are commonly considered to have made a major, if not essential, contribution to US vaccine coverage, the causal relations between mandates and population vaccination remain unclear owing to myriad cointerventions and 
confounding factors. For example, in cases in which US insurance companies have been reluctant to cover nonmandated vaccines (e.g., HPV vaccine for boys), mandates can increase vaccination rates by acting indirectly on insurers through school vaccine requirements. Therefore, implementing a mandate in a setting in which public coverage for vaccines is already universally offered may not result in an increase in coverage comparable to that seen in jurisdictions with more privatized coverage.

Policy-makers must consider many factors, including the variety of possibilities for exemptions, penalties and rewards, and how the mandate may be implemented, when weighing implementation of new or revised mandates - issues Attwell and colleagues ${ }^{5}$ classified as matters of severity and enforcement. Given the potential for a policy to fail to gain compliance, as seen with the US HPV mandates for girls, ${ }^{24,26,30,33,34,37}$ such issues are real and present in today's policy landscape. Our findings are largely congruent with those of Lee and Robinson, ${ }^{47}$ who found that, in most cases, childhood vaccination mandates through 2015 were associated with higher coverage in the US, with limited evidence of transferability to other settings. A review by the US Community Preventive Services Task Force that included studies published through $2012^{48}$ advised that interventions such as reminder systems and school-based vaccination clinics were cost-effective interventions to promote pediatric vaccine coverage ${ }^{49}$ and that such strategies to increase awareness of and access to vaccination could be attempted before proposing or strengthening a mandate.

\section{Limitations}

No search is exhaustive, so although we endeavoured to be systematic, transparent and comprehensive in our data collection and inclusion screening, it is possible we may have missed studies that may have been eligible for inclusion. At least 2 reviewers assessed for inclusion at every stage of review, but errors in judgment are possible. In addition, with nearly half of the included studies published within the past 3 years, there may be new studies currently under way that would contribute valuable information to our findings. Older studies, particularly those conducted before the mid-1990s, would have been conducted in an era of substantially different disease prevalence, vaccine products, vaccine schedules, data sources, data management practices and public health policies, which may limit the transferability of their findings to contemporary settings.

\section{Conclusion}

Adding well-accepted vaccines to an existing mandate, introducing a mandate in concert with reduction of structural barriers to vaccination or adding documentation requirements all appear to be associated with increased vaccination and/or documentation in most cases. It is unclear, however, to what extent such increases are due to the compulsory nature of the policies or to cointerventions that increase access and awareness. Education or vaccination mandates for highly politicized vaccines are more risky and may fail to be followed by the desired increase or even decrease uptake relative to nonmandate jurisdictions. To further the science of vaccination levers, highquality implementation research that uses whole-population coverage data and takes into consideration cointerventions, confounders, clustering of unvaccinated populations and context is required. Owing to the risk of backlash, in cases of highly politicized vaccines or jurisdictions without a tradition of mandates, other approaches such as improving access, education and documentation might be tried before moving to mandated vaccination.

\section{References}

1. Policy resolution GC15-C27: Declaration of immunization status. Ottawa: Canadian Medical Association; 2015 Aug. 26.

2. Immunization of School Pupils Act, 1990, R.S.O. 1990, c.I.1. Available: https:// www.ontario.ca/laws/statute/90i01 (accessed 2015 Dec. 18).

3. Public Health Act, 1998 (S.N.B. 1998, c.P-22.4). Available: www.ecolex.org/ details/legislation/public-health-act-snb-1998-c-p-224-lex-faoc095727/ (accessed 2017 Nov. 7).

4. Zussman R. B.C. planning on mandatory vaccination registration at schools by September, says health minister. Global News 2019 Feb. 26. Available: https:// globalnews.ca/news/5002410/mandatory-registration-vaccines-measles/ (accessed 2019 Mar. 2).

5. Attwell K, Navin MC, Lopalco PL, et al. Recent vaccine mandates in the United States, Europe and Australia: a comparative study. Vaccine 2018;36: 7377-84.

6. Gostin LO. Law, ethics, and public health in the vaccination debates: politics of the measles outbreak. FAMA 2015;313:1099-100.

7. Opel DJ, Omer SB. Measles, mandates, and making vaccination the default option. FAMA Pediatr 2015;169:303-4.

8. Blank NR, Caplan AL, Constable C. Exempting schoolchildren from immunizations: states with few barriers had highest rates of nonmedical exemptions. Health Aff (Millwood) 2013;32:1282-90.

9. Davis MM, Gaglia MA. Associations of daycare and school entry vaccination requirements with varicella immunization rates. Vaccine 2005;23:3053-60.

10. Omer SB, Pan WKY, Halsey NA, et al. Nonmedical exemptions to school immunization requirements: secular trends and association of state policies with pertussis incidence. $7 A M A$ 2006;296:1757-63.

11. Omer SB, Richards JL, Ward M, et al. Vaccination policies and rates of exemption from immunization, 2005-2011. NEngl f Med 2012;367:1170-1.

12. Pride KR, Geissler AL, Kolasa MS, et al. Assessment of vaccine exemptions among Wyoming school children, 2009 and 2011. F Sch Nurs 2014;30:332-9.

13. Thompson JW, Tyson S, Card-Higginson P, et al. Impact of addition of philosophical exemptions on childhood immunization rates. Am 7 Prev Med 2007; 32:194-201.

14. Yang Y'T, Debold V. A longitudinal analysis of the effect of nonmedical exemption law and vaccine uptake on vaccine-targeted disease rates. Am 7 Public Health 2014;104:371-7.

15. Liberati A, Altman DG, Tetzlaff J, et al. The PRISMA statement for reporting systematic reviews and meta-analyses of studies that evaluate health care interventions: explanation and elaboration. PLoS Med 2009;6:e1000100.

16. Higgins JPT, Green PS, editors. Cochrane handbook for systematic reviews of interventions. Version 5.1.0. Oxford (UK): Cochrane Collaboration; 2011. Available: www.handbook.cochrane.org (accessed 2019 June 17).

17. McGowan J, Sampson M, Salzwedel DM, et al. PRESS Peer Review of Electronic Search Strategies: 2015 guideline statement. 7 Clin Epidemiol 2016;75: 40-6.

18. Sterne JA, Hernán MA, Reeves BC, et al. ROBINS-I: a tool for assessing risk of bias in non-randomised studies of interventions. BMF 2016;355:14919.

19. Popay J, Roberts H, Sowden A, et al. Guidance on the conduct of narrative synthesis in systematic reviews: a product from the ESRC Methods Programme. Lancaster (UK): Lancaster University; 2006.

20. D'Ancona F, D'Amario C, Maraglino F, et al. Introduction of new and reinforcement of existing compulsory vaccinations in Italy: first evaluation of the impact on vaccination coverage in 2017. Euro Surveill 2018;23:1800238.

21. Thompson SC, Cocotsi L, Goudey RE, et al. An evaluation of school entry immunisation certificates in Victoria. Aust 7 Public Health 1994;18:267-73.

22. Omer SB, Allen K, Chang DH, et al. Exemptions from mandatory immunization after legally mandated parental counseling. Pediatrics 2018;141:e20172364.

23. Averhoff F, Linton L, Peddecord KM, et al. A middle school immunization law rapidly and substantially increases immunization coverage among adolescents. Am 7 Public Health 2004;94:978-84.

24. Bugenske E, Stokley S, Kennedy A, et al. Middle school vaccination requirements and adolescent vaccination coverage. Pediatrics 2012;129:1056-63.

25. Carpenter CS, Lawler EC. Direct and spillover effects of middle school vaccination requirements. Am Econ F Econ Policy 2019;11:95-125. 
26. Cuff RD, Buchanan T, Pelkofski E, et al. Rates of human papillomavirus vaccine uptake amongst girls five years after introduction of statewide mandate in Virginia. Am 7 Obstet Gynecol 2016;214:752.e1-6.

27. Jacobs RJ, Meyerhoff AS. Effect of middle school entry requirements on hepatitis B vaccination coverage. 7 Adolesc Health 2004:34:420-3.

28. Karikari Y, Akinyede O, Davis V, et al. Impact of vaccine mandate on Tdap vaccination coverage among Illinois students 2014-15. Pan Afr Med f 2017;27:103.

29. Morita JY, Ramirez E, Trick WE. Effect of a school-entry vaccination requirement on racial and ethnic disparities in hepatitis B immunization coverage levels among public school students. Pediatrics 2008;121:e547-52.

30. Moss JL, Reiter PL, Truong YK, et al. School entry requirements and coverage of nontargeted adolescent vaccines. Pediatrics 2016;138:e20161414.

31. Olshen E, Mahon BE, Wang S, et al. The impact of state policies on vaccine coverage by age 13 in an insured population. 7 Adolesc Health 2007;40:405-11.

32. Kharbanda EO, Stockwell MS, Colgrove J, et al. Changes in Tdap and MCV4 vaccine coverage following enactment of a statewide requirement of Tdap vaccination for entry into sixth grade. Am 7 Public Health 2010;100:1635-40.

33. Perkins RB, Lin M, Wallington SF, et al. Impact of school-entry and education mandates by states on HPV vaccination coverage: analysis of the 2009-2013 National Immunization Survey-Teen. Hum Vaccin Immunother 2016;12:1615-22.

34. Pierre-Victor D, Page TF, Trepka MJ, et al. Impact of Virginia's school-entry vaccine mandate on human papillomavirus vaccination among 13-17-year-old females. 7 Womens Health (Larchmt) 2017;26:266-75

35. Potter RC, DeVita SF, Vranesich PA, et al. Adolescent immunization coverage and implementation of new school requirements in Michigan, 2010. Am f Public Health 2014;104:1526-33.

36. Simpson JE, Hills RA, Allwes D, et al. Uptake of meningococcal vaccine in Arizona schoolchildren after implementation of school-entry immunization requirements. Public Health Rep 2013;128:37-45.

37. Thompson EL, Livingston MD, Daley EM, et al. Human papillomavirus vaccine initiation for adolescents following Rhode Island's school-entry requirement, 2010-2016. Am 7 Public Health 2018;108:1421-3.

38. Wilson TR, Fishbein DB, Ellis PA, et al. The impact of a school entry law on adolescent immunization rates. 7 Adolesc Health 2005;37:511-6.

39. Jackson CL, Carpenter RL. Effect of a state law intended to require immunization of school children. Health Serv Rep 1972;87:461-6.

40. Risk of bias tools - ROBINS-I template (2016). Available: https://sites. google.com/site/riskofbiastool/welcome/home/current-version-of-robins-i/ robins-i-template-2016 (accessed 2019 May 30).

41. Levin KA. Study design VI — Ecological studies. Evid Based Dent 2006; 7:108.

42. Sedgwick P. Ecological studies: advantages and disadvantages. BM7 2014; 348:g2979.

43. National Center for Immunization and Respiratory Diseases, Centers for Disease Control and Prevention. National Immunization Survey Teen: a user's guide for the 2016 public-use data file. Atlanta: Centers for Disease Control and Prevention; 2017. Available: www.cdc.gov/vaccines/imz-managers/nis/downloads/ NIS-TEEN-PUF16-DUG.pdf (accessed 2019 May 30).
44. Durlak JA, DuPre EP. Implementation matters: a review of research on the influence of implementation on policy outcomes and the factors affecting implementation. Am 7 Community Psychol 2008;41:327-50.

45. MacDonald NE, Harmon S, Dube E, et al. Mandatory infant \& childhood immunization: rationales, issues and knowledge gaps. Vaccine 2018;36: 5811-8.

46. Salmon DA, Teret SP, MacIntyre CR, et al. Compulsory vaccination and conscientious or philosophical exemptions: past, present, and future. Lancet 2006; 367:436-42.

47. Lee C, Robinson JL. Systematic review of the effect of immunization mandates on uptake of routine childhood immunizations. F Infect 2016;72:659-66.

48. Vaccination programs: requirements for child care, school, and college attendance. Atlanta: The Community Guide; 2016. Available: www.thecommunityguide.org/ findings/vaccination-programs-requirements-child-care-school-and-college-attendance (accessed 2018 Oct. 22).

49. Jacob V, Chattopadhyay SK, Hopkins DP, et al.; Community Preventive Services Task Force. Increasing coverage of appropriate vaccinations: a community guide systematic economic review. Am 7 Prev Med 2016;50:797-808.

Affiliations: Department of Communication (Greyson), University of Massachusetts, Amherst, Mass.; Vaccine Evaluation Center (Greyson, Bettinger), BC Children's Hospital Research Institute; Department of Pediatrics (Greyson, Bettinger) and School of Library, Archival and Information Studies (Vriesema-Magnuson), University of British Columbia, Vancouver, BC

Contributors: Devon Greyson and Julie Bettinger conceived of the study. Chris Vriesema-Magnuson and Devon Greyson conducted the literature searches. Devon Greyson drafted the manuscript. All of the authors analyzed the data, critically revised the manuscript for important intellectual content, approved the final version to be published and agreed to act as guarantors of the work.

Funding: Funding for this study was provided by the British Columbia Immunization Committee. Julie Bettinger is supported by a Michael Smith Foundation for Health Research Scholar Award.

Acknowledgements: The authors thank the peer reviewers, including the 2 CMAf Open reviewers, who strengthened the manuscript, and Alex Goudreau, who provided Peer Review of Electronic Search Strategies (PRESS) for the MEDLINE search strategy.

Supplemental information: For reviewer comments and the original submission of this manuscript, please see www.cmajopen.ca/content/7/3/ E524/suppl/DC1. 